\title{
Synchronization and persistence in Diffusively Coupled Lattice Oscillators
}

\author{
Adu A.M. Wasike* and K.T. Rotich
}

School of Mathematics, University of Nairobi,P.O. Box 30197-00100, Nairobi, Kenya, *Email: aduwasike@yahoo.com.

\section{النوايق والثبلت في النشار اللثبكة المزنوجة للمنببلت}

\author{
ألو أم. ولهيك وكت. روتيث
}

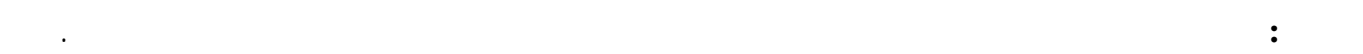

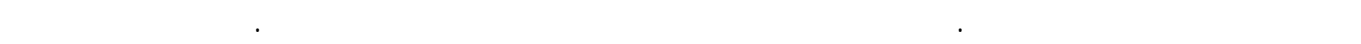

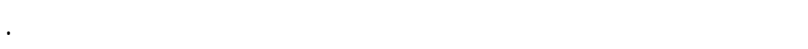

\begin{abstract}
We consider the synchronization and persistence of a system of identical lattice oscillators that are diffusively coupled to their nearest neighbours. Each subsystem has a compact global attractor. This is done in the framework of invariant manifold theory. Normal hyperbolicity and its persistence are applied to obtain general conditions for the stability and robustness of the synchronization manifold.
\end{abstract}

AMS(MOS) Subject classifications: 37C80, 37D10.

KEYWORDS: Lattice oscillators, bravais lattice, synchronization manifold, robustness.

\section{Introduction}

$\mathbf{T}$

There has been great interest in the synchronization of coupled oscillators on a one dimensional integer lattice. Aside from the mathematical interest in the problem, coupled oscillators occur in physics, engineering, communications, signal processing and many other areas see for instance (see for instance Afraimovich et al.(1992), Cuomo (1993) and Oppenheim (1993), and references therein. In electrical circuit theory, much work is due to Chua and Roska (1993), in particular in the studies of cellular neural networks (CNN). Metallurgy is another area where lattice systems are found ( see for instance Cahn (1960)). Basic to the study of synchronization, two fundamental questions are of interest. The first is to do with the stability of the synchronization state of the system and the second is its robustness. Robustness of the synchronization state is its ability to be insensitive to small perturbations in the system that generates it. For coupled identical systems, the diagonal of the system is invariant. Synchronization is equivalent to the attracting property of the diagonal, which in turn is determined by the Lyapunov exponents normal to the diagonal. More specifically, if all the Lyapunov exponents normal to the diagonal are negative, then the coupled oscillators are synchronized. In 
practice, for instance in the implementation of some model, various perturbations are unavoidable. Moreover, numerical computations of model equations deal with solving "perturbed models". For this reason, it is natural to address the question of not only the stability of the synchronization manifold but also its persistence or rather robustness under perturbations.

Much of the work on synchronization is on one dimensional lattice and deals mainly with its stability (see for instance the classical paper of Fujisaki and Yamada (1983)).

Normal hyperbolicity and the generalized Lyapunov exponents have been used to establish conditions for the stability and persistence of synchronization manifold for two dissipative systems each with a compact global attractor (see for instance Chow and Liu (1997), Josića (1977)). There is less repertoire on this subject in the two- and three- dimensional lattices. The problem we consider in this paper therefore, is concerned with the synchronization, stability, and persistence of oscillations of a system of ordinary differential equations (ODE's) indexed by points in an $m$-dimensional integer lattice $z_{+}^{m}, m \in\{1,2\}$. This system is what we shall refer to as lattice differential equations (LDE's). By a lattice oscillator, we mean a system of LDE's with every lattice point having an attracting periodic orbit ( see for instance Chow and Liu (1997), Wasike (2003)). We look at these two phenomena for oscillators that are linearly and symmetrically coupled via a diffusion-like coupling to their nearest neighbours on $m$-dimensional lattices.

This is how we approach this problem. We set the problem in the framework of dynamical systems and then consider the two aspects of synchronization based on invariant manifold theory. In particular, we shall apply the ideas of generalized Lyapunov exponents and normal hyperbolicity as defined in Fenichel (1971), and Hirsch et al. (1977) respectively. This approach will enable us to compare the rate of growth in the transversal direction to the synchronization manifold and that along the manifold. We first look at the one-dimensional lattice, then proceed to the two-dimensional case. Most of the definitions basic terminology and some results will be given in $\S 2$ while $\S 3$ deals with a two dimensional lattice. In every case, we give conditions that guarantee synchronization of oscillators and the stability of synchronization manifold under perturbation. We also give conditions under which the synchronization manifold is robust. $\$ 4$ is the conclusion.

Let us suppose that the dynamics at every lattice site $i$ is governed by the system of ODEs

$$
\dot{z}_{i}=g\left(z_{i}\right)
$$

where the dot "“" denotes differentiation with respect to time $t, z_{i}=z_{i}(t) \in \mathrm{R}^{d}, d \geq 2, i=1, \ldots . ., n$, the number of oscillators, $g \in C^{r}\left(\mathrm{R}^{d}, \mathrm{R}^{d}\right), r \geq 1$.

Suppose that, for each $i$, there is a compact global attractor for Equation (1); that is, there exist a compact set which is invariant under the flow defined by Equation (1) and the $\omega$-limit set for each orbit of Equation (1) belongs to this set.

We shall now couple these systems to obtain one- and two-dimensional lattices.

\section{One dimensional lattice}

Now let us couple the $n \geq 2$ identical subsystems in Equation (1) to yield

$$
\dot{z}=B(k) z+f(z)=: X(z, k),
$$

where $z=\left(z_{1}, z_{2}, \ldots \ldots, z_{n}\right)^{T}$ with $T$ denoting transpose, $z_{i}$ denotes the coordinates of a point on the lattice, $B(k)$ a real symmetric matrix depending on the coupling strength $k \geq 0$, describes the coupling configuration. If we consider symmetric nearest neighbour diffusive coupling on a linear lattice $(m=1)$ with Neumann 


\section{DIFFUSIVELY COUPLED LATTICE OSCILLATORS}

boundary conditions and assume that oscillators influence each other equally ( $k=$ constant), then $B(k)=k \Delta_{1} \otimes I_{d}$ with

$$
\Delta_{1}=\left(\begin{array}{cccccccc}
-1 & 1 & 0 & 0 & \cdots & 0 & 0 & 0 \\
1 & -2 & 1 & 0 & \cdots & 0 & 0 & 0 \\
0 & 1 & -2 & 1 & \cdots & 0 & 0 & 0 \\
\vdots & \vdots & \vdots & \vdots & \ddots & \vdots & \vdots & \vdots \\
0 & 0 & 0 & 0 & \cdots & 1 & -2 & 1 \\
0 & 0 & 0 & 0 & \cdots & 0 & 1 & -1
\end{array}\right) \in \mathrm{R}^{n \times n}
$$

$I_{d}$ a $d$ x $d$ identity matrix, $f(z)=\left(g\left(z_{1}\right), g\left(z_{2}\right), \ldots \ldots, g\left(z_{n}\right)\right)^{T} \in C^{r}\left(\mathrm{R}^{n d}, \mathrm{R}^{n d}\right)$; and $\otimes$ is a

Kronecker product.

Definition 1. Equation (2) is symmetrically synchronized if there exists a compact diagonal-like, smooth dmanifold $M_{k}$ that belongs to the manifold

$$
M_{1}=\left\{z \in R^{n d}: z_{1}=z_{2}=\ldots=z_{2} \neq 0\right\} .
$$

$M_{1}$ has a boundary and is inflowing invariant and locally attracting.

We refer to this set as the "diagonal" or the synchronization manifold in $R^{\text {nd }}$, (see for instance Hale (1997)). For the system to be synchronized, the diagonal $M_{1}$ must be invariant and attracting under the flow defined by Equation (2). In many applications, we are usually interested in local synchronization; that is, instead of requiring global attraction of $M_{1}$, we seek for local attraction. In this case we will say that the system in question is locally symmetrically synchronized.

Let us suppose that System (2) has a compact global attractor $M_{k}$ for each $k$. We say that Equation (2) is symmetrically synchronized if its solution $z$ is in the set $M_{1}$ for all $t \geq 0$; that is, $M_{k} \subset M_{1}$. $M_{k}$ needs to persist under perturbation for synchronization to be of physical interest.

Definition 2. Let $X(z, k)$ be the vector field defined in Equation (2) and $\tilde{X}(t, k)$ be its perturbation, then; synchronization manifold $M_{k}$ is $C^{1}$ stable or persistent if for any $\in>0$, there exist $\delta>0$ such that for any $|\tilde{X}(z, k)-X(z, k)|_{C^{1}}<\delta$ and the system

$$
\dot{\tilde{z}}(t)=\tilde{X}(z, k)
$$

is locally synchronized with the synchronization manifold $\tilde{M}_{k}$ such that

$$
\left|\tilde{M}_{k}-M_{k}\right|_{C^{1}}<\in \text {. }
$$


We say that the synchronization manifold is Robust if $\in \ll 1$.

Very often in the study of synchronization, systems have a natural invariant submanifold. A necessary and sufficient condition for such synchronization and persistence is normal hyperbolicity Mané (1977). The manifold $M_{k}$ is normally hyperbolic if, under the dynamics linearized about the invariant manifold, the growth (decay) rate of vectors transverse to the manifold dominates the growth (decay) rate of the vectors tangent to the manifold, that is; the expansion (contraction) of the flows in the direction normal to the manifold is exponentially greater than the expansion (contraction) of the flows tangent to the manifold (see for instance Hirsch et al. (1977), Mané (1977)).

The growth/decay rates of vectors can be characterized in terms of Generalised Lyapunov exponents determined from the linearized equations of motion around the synchronization manifold (see for instance Fenichel (1971), Wiggins (1994)). Let us make this more precise.

Consider the linearization of Equation (2) along the manifold $M_{k}$

$$
\dot{z}=A\left(z\left(t ; z_{0}\right)\right) z
$$

where $z\left(t ; z_{0}\right)$ is the solution of Equation (2) with $z\left(0 ; z_{0}\right)=z_{0} \in M_{k} \quad A(z)=J f(z)$ is the Jacobian matrix of $f$ at $z$. Assume there is an invariant splitting of the fundamental matrix solution of Equation (4), $\Phi\left(t ; z_{0}\right)$; that is, $T_{z} R^{n d}=T_{z} M_{k} \oplus N_{z}$ for $z \in M_{k}, \Phi\left(t ; z_{0}\right) T_{z_{0}} M_{k}=T_{z\left(t, z_{0}\right)} M_{k}$, and $\Phi\left(t ; z_{0}\right) N_{z_{0}}=N_{z\left(t, z_{0}\right)}$ for all and $t \in R^{1}$. Let $\Phi_{C}\left(t ; z_{0}\right)$ and $\Phi_{S}\left(t ; z_{0}\right)$ denote the restrictions of $\Phi\left(t ; z_{0}\right)$ on $T_{z_{0}} M_{k}$ and $N_{z_{0}}$ respectively, where $T_{z_{0}} M_{k}$ and $N_{z_{0}}$ are the tangent and normal vectors to $M_{k}$ at $z_{0}$ respectively and $\oplus$ indicates the direct sum of the two vector spaces.

Definition 3. The generalized Lyapunov exponents for $z_{0} \in M_{k}$ are defined as

$$
\begin{gathered}
\alpha\left(z_{0}\right)=\limsup _{t \rightarrow \infty} \frac{1}{t} \ln \left\|\Phi_{s}\left(t ; z_{0}\right)\right\|, \\
\beta\left(z_{0}\right)=\limsup _{t \rightarrow \infty} \frac{\ln \left\|\Phi_{s}\left(t ; z_{0}\right)\right\|}{\ln m\left(\Phi_{C}\left(t ; z_{0}\right)\right)},
\end{gathered}
$$

where, for a linear operator $L, m(L)=\min \{|L x|:|x|=1, x \in D(L)\}, D(L)$ is the domain of the operator $L$.

The generalized Lyapunov exponents have been used in the study of normally hyperbolic invariant manifolds (see Hirsch et al. (1977), Fenichel (1971), Chow and Liu(1997)). The uniformity Lemma in Fenichel (1971) states that $\alpha$ and $\beta$ achieve their maximums on $M_{k}$. For this reason we need to show that there exists an invariant manifold $M_{k}$ in the solution space of Equation (2). We describe this manifold in appropriate coordinates that display vectors transverse and tangent to it. Since we are considering coupled identical oscillators, the diagonal is invariant. We therefore show that the matrix $\Delta_{1}$ has zero as an eigenvalue with the 


\section{DIFFUSIVELY COUPLED LATTICE OSCILLATORS}

corresponding generalized eigenspace that belongs to the diagonal in $R^{\text {nd }}$ and the other eigenvalues are bounded to the left-hand side of the complex plane.

As a consequence of the preceding statement, the following hypotheses about $B(k)$ are made:

$\mathbf{H}_{\mathbf{1}}$ For each $k, B(k)$ is self adjoint, $\lambda_{0}=0$ is an eigenvalue of $B(k)$ with the corresponding generalized eigenvectors $e, \quad \operatorname{vec}(1,1, \ldots \ldots . . .1) \in R^{\text {nd }}$ whose span is the diagonal in $R^{\text {nd }}$.

$\mathbf{H}_{2}$ For $n>2$, there exist a $k_{0}$ and a bounded set $U \in R^{n d}$ such that for each $k>k_{0}$, Equation (2) has a global attractor $A_{k} \in U$.

Let us now show that Equation (2) satisfies the two hypotheses. Clearly the eigenvalues of $B(k)$ are $\lambda_{0}=0$ and $\lambda_{s}=-k\left(2+2 \cos \frac{s \pi}{n}\right), s=1,2, \ldots . n-1 \quad$ (see for instance Lancaster and Tismenetsky (1995)). Hence $\mathbf{H}_{1}$ is satisfied. For $\mathbf{H}_{2}$ we have to make a coordinate transformation that we now describe.

From $\mathbf{H}_{1}$ we can introduce a new coordinate system

$$
\begin{gathered}
z=y e+\tilde{e} w, w=\left(w_{1}, w_{2}, \ldots . . w_{n-1}\right)^{T}, w \\
w_{j}=\mathrm{R}_{j}^{n d-d}, y \in n d-\mathrm{R}^{d+1}, 1 \leq j \leq n-1, \\
y=\frac{1}{n} \sum_{j=1}^{n} z_{j},
\end{gathered}
$$

where $e_{j}$ is the usual unit vector in $\mathrm{R}^{n}$ with zeros except for 1 at the $\mathrm{j}^{\text {th }}$ position and $\tilde{e}_{j}=\sum_{i=1}^{j} e_{i}-\frac{j}{n} e$, with $\tilde{e}=\left(\tilde{e}_{1}, \tilde{e}_{2}, \ldots . ., \tilde{e}_{n-1}\right)$. The set $e, \tilde{e}_{j}, 1 \leq j \leq n-1$ is an orthogonal basis for $\mathrm{R}^{n}$. Using transformation in (7) in Equation (2), we obtain

$$
\begin{aligned}
& \dot{w}=k\left(\Delta \otimes I_{d}\right) w+F(w, y), \\
& \dot{y}=\frac{1}{n} \sum_{j=1}^{n} g\left(z_{j}\right),
\end{aligned}
$$

where the function $F(w, y)=\left(F_{1}(w, y), F_{2}(w, y), \ldots \ldots, F_{n-1}(w, y)\right)^{T}$ with $F_{j}(w, y)=\left(g\left(z_{j}\right)-g\left(z_{j+1}\right)\right), 1 \leq j \leq n-1$, and the matrix $\Delta$ is given by 


$$
\Delta=\left(\begin{array}{rccccccc}
-2 & 1 & 0 & 0 & \cdots & 0 & 0 & 0 \\
1 & -2 & 1 & 0 & \cdots & 0 & 0 & 0 \\
0 & 1 & -2 & 1 & \cdots & 0 & 0 & 0 \\
\vdots & \vdots & \vdots & \vdots & \ddots & \vdots & \vdots & \vdots \\
0 & 0 & 0 & 0 & \cdots & 1 & -2 & 1 \\
0 & 0 & 0 & 0 & \cdots & 0 & 1 & -2
\end{array}\right) \in \mathrm{R}^{(n-1) \times(n-1)}
$$

The eigenvalues of $\Delta$ are $\lambda_{s}=-2-2 \cos \frac{s \pi}{n}, s=1,2,3, \ldots . ., n-1$, (see for instance Lancaster and Tismenetsky (1995)). Since the matrix $\Delta_{1}$ has zero as an eigenvalue and other eigenvalues are bounded to the left-hand side of the complex plane, Equation (8) satisfies $\mathbf{H}_{\mathbf{1}}$ and $\mathbf{H}_{\mathbf{2}}$ hence there exists an invariant manifold $M_{k}$ of Equation (8) containing the attractor $A_{k}$ and attracting bounded sets of $\mathrm{R}^{\text {nd }}$ (see Hale (1997)). As the coordinate transformation in Equation (7) is linear, it follows that Equation (2) satisfies $\mathbf{H}_{2}$.

The first equation in (8) describes the motion transverse to the synchronization manifold $M_{k}$; that is, it describes the deviations $z_{j}-z_{j+1}$. Synchronization means that the deviations dampen out as $t \rightarrow \infty$. From the first equation in (8), this is equivalent to saying that $\{w=0\}$ is exponentially stable. Since we are interested in local synchronization, the criteria of local attractivity of the synchronization manifold and its persistence is determined by the ratio of the growth rates of vectors transverse and tangential to it. The following theorem relates robustness and Lyapunov exponents.

\subsection{Main results}

In this section, we state and proof a theorem that relates robustness and Lyapunov exponents. For this purpose, we shall need some results due to Chow and Liu (1997). These results make use of the well-known theorems in invariant manifold theory regarding persistence of invariant manifolds, (see for example, Fenichel (1971), Hirsch et al. (1977), and Mané (1977)) and they are stated thus:

Lemma 1 (Chow and Liu (1997)). Consider Equation (2) and suppose that the synchronization manifold $M_{k}$ is invariant, then if $\alpha\left(z_{0}\right)<0$, for all $z_{0} \in M_{k}$, then the manifold $M_{k}$ is attracting and hence (2) is synchronized.

Lemma 2 (Chow and Liu (1997)). Suppose that $M_{k}$ is locally synchronized, then the synchronization is $C^{1}$ table if and only if $\alpha\left(z_{0}\right)<0$ and $\beta\left(z_{0}\right)<1$ for all $z_{0} \in M_{k}$.

Theorem 3. If Equation (2) satisfies $\mathbf{H}_{1}$ and $\mathbf{H}_{2}$, then there exist a $k_{0}$ such that for all $k>k_{0}$, there is a positively invariant synchronization manifold $M_{k}$ that is attracting and $C^{1}$ stable or persistent.

Proof. Characterizing growth rates in the fashion described above requires knowledge of the linearized dynamics near orbits on the invariant manifold as $t \rightarrow \infty$. 


\section{DIFFUSIVELY COUPLED LATTICE OSCILLATORS}

Linearization of (8) along the solution $\left(0, y_{0}(t)\right)$ which corresponds to $(w(t), y(t)) \in M_{k}$ yields

$$
\left(\begin{array}{c}
\dot{w} \\
\dot{y}
\end{array}\right)=\left(\begin{array}{cc}
I_{n-1} \otimes D_{z} g\left(y_{0}(t)\right)+k \Delta \otimes I_{d} & 0 \\
0 & D_{z} g\left(y_{0}(t)\right)
\end{array}\right)\left(\begin{array}{l}
w \\
y
\end{array}\right),
$$

Where $D_{z} g\left(y_{0}(t)\right)=J g\left(y_{0}(t)\right)$ is the Jacobian matrix of $g$ at $y_{0}(t)$ the solution of Equation (1).

Notice that there is a continuous invariant splitting of $T \mathrm{R}^{n d}\left|M_{k} ; T \mathrm{R}^{n d}\right| M_{k}=T M_{k} \oplus N$, where $\oplus$ refers to the Whitney sum of the tangent and normal bundles. Since System (9) is uncoupled and $I_{n-1} \otimes D_{z} g\left(y_{0}(t)\right)$ and $k \Delta \otimes I_{d}$ commute, the fundamental matrix solution of Equation (9) is of the form

$$
\left(\begin{array}{l}
w(t) \\
y(t)
\end{array}\right) \approx\left(\begin{array}{cc}
e^{\left(k \lambda_{S}+\lambda_{l}\right) t} & 0 \\
0 & e^{\lambda_{l} t}
\end{array}\right):=\Phi\left(t: z_{0}\right),
$$

where $\lambda_{s}, 1 \leq s \leq n d-d$ are the eigenvalues of the coupling matrix $\Delta \otimes I_{d}$ and $\lambda_{l}, 1 \leq l \leq d$, are the Lyapunov exponents of the trajectories defined by $\dot{y}=D_{z} g\left(y_{0}(t)\right) y$.

The invariant manifold $\{w=0\}$ is generated by the second equation in (9). Therefore $\Phi_{c}\left(t, z_{0}\right)$ corresponds to $e^{\lambda_{l} t}$ while $\Phi_{S}\left(t, z_{0}\right)$ corresponds to $e^{\left(k \lambda_{S}+\lambda_{l}\right) t}$.

The maximum eigenvalue of $\Delta$ is $\lambda_{1}=-4 \sin ^{2} \frac{\pi}{2 n}$ and hence

$$
\alpha\left(z_{0}\right):=\left(-k \zeta_{1}+\lambda_{M}\right)
$$

where $\zeta_{1}:=-\lambda_{1}$ is the maximum eigenvalue of the coupling configuration and $\lambda_{M}$ is the maximum Lyapunov exponent defined by $\dot{y}=D_{z} g\left(y_{0}(t)\right) y$ in $M_{k}$. Thus by Lemma $1,\{w=0\}$ is attracting and the coupled system is locally synchronized if $\alpha\left(z_{0}\right)<0$; that is, $k$ satisfies $k>\frac{1}{\xi_{1}} \lambda_{M}$. To insure robustness of the synchronization manifold, the condition for normal hyperbolicity must be satisfied. $M_{k}$ is robust if and only if for all $z_{0} \in M_{k}$,

$$
\sup _{z_{0}}\left\|\Phi_{S}\left(t, t_{0}\right)\right\|<\inf _{z_{0}} m\left(\Phi_{C}\left(t, t_{0}\right)\right)
$$

(see for instance Hirsch et al. (1977) page 10). This is equivalent to saying that

$$
\beta\left(z_{0}\right):=\limsup _{t \rightarrow \infty} \frac{\ln \left\|\Phi_{s}\left(t, z_{0}\right)\right\|}{\ln m\left(\Phi_{C}\left(t, z_{0}\right)\right)}<1 .
$$


By Lemma 2, we require that $\alpha\left(z_{0}\right)<0$ and $\beta\left(z_{0}\right)<1$; that is,

$$
\frac{\lambda_{M}-k \zeta_{1}}{\lambda_{m}}<1
$$

where $\lambda_{m}$ is the minimum Lyapunov exponent defined by $\dot{y}=D_{z} g\left(y_{0}\right) y$ in $M_{k}$. This is satisfied if $k>\frac{1}{\zeta_{1}}\left(\lambda_{M}-\lambda_{m}\right)$. Thus we conclude that for synchronization, stability and robustness of the synchronization manifold $M_{k}, k$ must satisfy the inequality

$$
k>\max \left\{\frac{1}{\zeta_{1}}\left(\lambda_{M}, \lambda_{M}-\lambda_{m}\right)\right\}
$$

Taking $k_{0}=\max \left\{\frac{1}{\zeta_{1}}\left(\lambda_{M}, \lambda_{M}-\lambda_{m}\right)\right\}$ completes the proof.

\section{Two dimensional lattice oscillators}

In this section we study the existence and stability of the synchronization manifold and its persistence. Results similar to those of one dimensional case can be shown to hold. All that one needs is to show that a synchronization manifold exists and make appropriate coordinate transformation which decomposes the flow along the invariant manifold into transversal and tangential flows and study the condition of normal hyperbolicity using generalized Lyapunov exponents.

Let us consider an $n \times n$ simple (or Bravais) square lattice. Let $z_{i j} \in \mathrm{R}^{d}, 1 \leq i, j \leq n$, be the coordinate of the $(\mathrm{i}, \mathrm{j})^{\text {th }}$ site oscillator and the matrix $\mathrm{Z}:=\left[\mathrm{Z}_{i j}\right]_{i, j=1}^{n} \in \mathrm{R}^{\text {nd } \mathrm{x} n d}$. Also let $\mathrm{Z}=\left(Z_{1}, Z_{2}, \ldots \ldots \ldots . ., Z_{n}\right)$ with $Z_{r} \in \mathrm{R}^{n d}, 1 \leq r \leq n$ be its $\mathrm{r}^{\text {th }}$ column. We therefore define the vector valued function of $\mathbf{Z}$ as

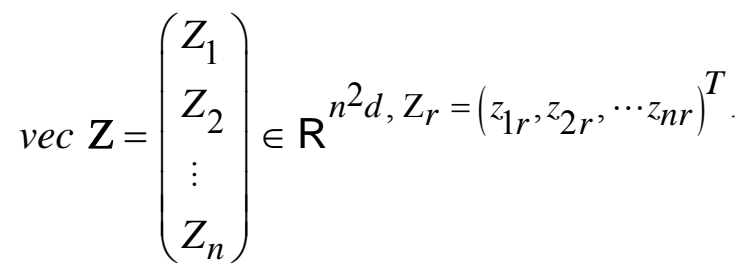

We also define the Kronecker sum of $\Delta_{1}$ with itself as:

$$
\Delta_{1} \oplus \Delta_{1}=\Delta_{1} \otimes I_{n}+I_{n} \otimes \Delta_{1},
$$

(see, for example Lancaster and Tismenetsky(1985) for details on matrix tensor algebra).

Let us couple the subsystems $\dot{z}_{i j}=g\left(z_{i j}\right)$ thus:

$$
\dot{\mathrm{Z}}=k\left[\left(\Delta_{1} \otimes I_{d}\right) \mathrm{Z}+\mathrm{Z} \Delta_{1}\right]+G(\mathrm{Z}),
$$




\section{DIFFUSIVELY COUPLED LATTICE OSCILLATORS}

where

$$
\mathbf{G}(Z):=\left[g\left(z_{i j}\right)\right]_{i j=1}^{n}=\left(f\left(Z_{1}\right), f\left(Z_{2}\right), \ldots \ldots . . f\left(Z_{n}\right)\right) .
$$

This type of coupling corresponds to symmetric nearest neighbour diffusive coupling on a simple square lattice with Neumann boundary conditions.

Writing Equation (12) in vector notation we obtain:

$$
\text { vec } \dot{\mathrm{Z}}=\mathrm{B}(k) \text { vec } \mathrm{Z}+\text { vec } G(\mathrm{Z}) \text {, }
$$

where the matrix $\mathrm{B}(k):=k\left(\Delta_{1} \oplus \Delta_{1}\right) \otimes I_{d}$.

For System (13) to be synchronized, the diagonal must be an invariant set which will be the case if all the $g\left(z_{i j}\right)$ are the same, and $\mathrm{B}(k)$ has zero as an eigenvalue with the diagonal as the corresponding generalized eigenspace. In this situation, we must also have all other eigenvalues of $\mathrm{B}(k)$ less than zero. We therefore make the following assumptions on $\mathrm{B}(k) . \mathbf{H}_{3}$ For each $k, \mathrm{~B}(k)$ is self adjoint, $\lambda_{0}=0$ is an eigenvalue of $\mathrm{B}(k)$ with the corresponding generalized eigenvectors $e \triangleq v e c(1,1, \ldots \ldots ., 1) \in \mathrm{R}^{n^{2} d}$ whose span is the diagonal in $\mathrm{R}^{n^{2} d} . \mathbf{H}_{4}$ For $n>2$, there exists a $k_{0}$ and a bounded set $U \in \mathrm{R}^{n^{2} d}$ such that for each $k>k_{0}$, Equation (13) has a global attractor $A_{k}^{2} \in U$.

It is trivial to show that Equation (13) satisfies $\mathbf{H}_{3}$. Symmetric synchronization occurs when $\mathrm{B}(k) \operatorname{vec} \mathrm{Z}=\lambda \operatorname{vec} \mathrm{Z}=0, \mathrm{Z} \neq 0$. Thus we require that $\lambda=0$ be an eigenvalue of $\mathrm{B}(k)$. Indeed the eigenvalues of $\mathrm{B}(k)$ are

$$
\sigma(\mathrm{B}(k)):=\left\{\mu_{s p} \mid \mu_{s p}=\left(\lambda_{s}+\lambda_{p}\right) k, 0 \leq s, p \leq n-1\right\},
$$

with

$$
\lambda_{0}=0, \lambda_{\xi}=-2-2 \cos \frac{\xi \pi}{2 n}, \xi=s, p, 1 \leq \xi \leq n-1
$$

each occurring $d$ times, the order of $I_{d}$. The corresponding eigenvector to $\lambda=0$ is given by $V:=\operatorname{col}(1,1, \ldots \ldots \ldots, 1) \in \mathbf{R}^{n^{2} d}$. The synchronization manifold corresponds to the $\operatorname{span}\{V\}$ that we define as follows:

$$
M_{1}^{2}:=\left\{\mathbf{Z} \mid Z_{j}=Z_{j+1}, Z_{j} \in M_{1}, 1 \leq j \leq n-1\right\}
$$

Thus $\mathbf{H}_{3}$ is satisfied. To proof that $\mathbf{H}_{4}$ is satisfied, we need to make a coordinate transformation. The transformation used in one dimensional case can be rewritten to suit the two dimensional case as follows.

$$
\begin{aligned}
& W_{j}=Z_{j}-Z_{j+1}, 1 \leq j \leq n-1, Z_{j} \in \mathrm{R}^{n d} \\
& Y=\frac{1}{n} \sum_{j=1}^{n} Z_{j}, Y \in \mathrm{R}^{n d}
\end{aligned}
$$


where $Y=\left(y_{1}, y_{2}, \ldots \ldots \ldots ., y_{n}\right)^{T}$, with $y_{i}=\sum_{j=1}^{n} z_{i, j}$.

Let $\tilde{e}$ be as defined in $\S 2$, and $e_{j}$ be the usual unit vector in $\mathrm{R}^{n}$. The set $e, \tilde{e}_{j}, 1 \leq j \leq n-1$ is an orthogonal basis for $\mathrm{R}^{n}$, and in this basis we can write $\mathbf{Z}$ as

$$
\text { vec } \mathbf{Z}=e \otimes Y+\left(\tilde{e} \otimes I_{n}\right) \text { vec } \mathbf{W}, \mathbf{W} \in \mathrm{R}^{n^{2} d-n d},
$$

where $\mathbf{W}:=\left(W_{1}, W_{2}, \ldots \ldots \ldots, W_{n-1}\right)$ with $W_{j} \in \mathrm{R}^{n d}, 1 \leq j \leq n-1$ as its $\mathrm{r}^{\text {th }}$ column.

Using Equations (14) and (15), Equation (13) satisfies

$$
\begin{aligned}
& \text { vec } \dot{\mathbf{W}}=k\left[\left(\Delta_{1} \oplus \Delta\right) \otimes I_{d}\right] \text { vec } \mathbf{W}+\operatorname{vec} \tilde{F}(\mathbf{W}, Y), \\
& \dot{Y}=k \Delta_{1} \otimes I_{d} Y+\frac{1}{n} \sum_{j=1}^{n} f\left(Z_{j}\right),
\end{aligned}
$$

where $\tilde{F}(\mathbf{W}, Y)=\left(\tilde{F}_{1}(\mathbf{W}, Y), \ldots \ldots, \tilde{F}_{n-1}(\mathbf{W}, Y)\right)$, with $\tilde{F}_{j}(\mathbf{W}, Y)=f\left(Z_{j}\right)-f\left(Z_{j+1}\right)$.

Since the matrix $\mathrm{B}(k)$ has zero as an eigenvalue and other eigenvalues bounded to the left hand side of the complex plane, Equation (13) satisfies $\mathbf{H}_{3}$ and $\mathbf{H}_{4}$ hence there exists an invariant manifold $M_{k}^{2}$ of Equation (13) containing the attractor $A_{k}^{2}$ and attracting sets bounded sets of $\mathrm{R}^{n^{2} d}$ ( see Hale (1997)). The first equation in (16) describes the transverse motion to the synchronization manifold, $M_{k}^{2}$, and the second equation describes the motion tangent to the manifold $M_{k}^{2}$. Symmetric synchronization is equivalent to $z_{p, q}-z_{r, s} \rightarrow 0$ as $t \rightarrow \infty$, for all $p, q, r, s$. This means that $W \rightarrow 0$ as $t \rightarrow \infty$. This implies that the synchronization manifold is attracting.

\subsection{Synchronization and persistence of $M_{k}^{2}$}

Since our interest is local synchronization of the invariant manifold, we shall seek to show that the manifold is locally attracting. The manifold $M_{k}^{2}$ is attracting if the Lyapunov exponents of the flow defined by the first equation in Equation (16) are less than zero.

Theorem 4. If System (13) satisfies $\mathbf{H}_{3}$ and $\mathbf{H}_{4}$, then there exists a $k_{0}$ such that for all $k>k_{0}$, there is a positively invariant synchronization manifold $M_{k}^{2}$ that is attracting and $C^{1}$ stable or persistent.

Proof. Linearization of (16) along $M_{k}^{2}$; that is, $(\mathbf{W}(t), Y(t))=\left(0, Y_{0}(t)\right)$, where $\left.Y_{0}(t):=\left(y_{01}(t)\right), y_{02}(t), \ldots \ldots, y_{0 \mathrm{n}}(t)\right)$, a solution to Equation (12), gives 


$$
\left(\begin{array}{c}
\dot{v} e c \mathbf{W} \\
\dot{Y}
\end{array}\right)=\left(\begin{array}{cc}
k\left(\Delta_{1} \oplus \Delta\right) \otimes I_{d}+I_{n-1} \otimes D G\left(Y_{0}(t)\right) & 0 \\
0 & D G\left(Y_{0}(t)\right)
\end{array}\right)\left(\begin{array}{c}
\operatorname{vec} \mathbf{W} \\
Y
\end{array}\right)
$$

where $D G\left(Y_{0}(t)\right):=I_{n} \oplus \operatorname{diag} D_{z} g\left(y_{0}(t)\right)$, is the is the Jacobian matrix of $f$ at $Y_{0}(t)$.

Notice that there is a continuous invariant splitting of $T \mathrm{R}^{n^{2} d}, T \mathrm{R}^{n^{2} d} \mid M_{k}^{2}=T M_{k}^{2} \oplus N$, where $T M_{k}^{2} \in \mathrm{R}^{n^{2} d-n d}$ and $N \in \mathrm{R}^{n d}$. From Lemma $1, \mathbf{W}=\mathbf{0}$ is locally synchronized if the maximum Lyapunov exponent of the trajectories defined by the first equation in Equation (17) are negative. Since Equation (17) is uncoupled, and the matrices $k\left(\Delta_{1} \oplus \Delta\right) \otimes I_{d}$ and $I_{n-1} \otimes D G\left(Y_{0}(t)\right)$ commute, the fundamental matrix solution of Equation (17) is of the form

$$
\left(\begin{array}{c}
\operatorname{vec} \mathbf{W}(t) \\
Y(t)
\end{array}\right) \approx\left(\begin{array}{cc}
e^{\left(k \hat{\lambda}_{\zeta}+\lambda_{i}\right) t} & 0 \\
0 & e^{\lambda_{i} t}
\end{array}\right):=\hat{\Phi}\left(t ; z_{0}\right),
$$

where $k \hat{\lambda}_{\zeta}, 1 \leq \zeta \leq n^{2} d-n d \quad$ are the eigenvalues of $k\left(\Delta_{1} \oplus \Delta\right), \lambda_{i}, 1 \leq i \leq n^{2} d-n d \quad$ and $\lambda_{i}, 1 \leq j \leq n d$ are the Lyapunov exponents over $M_{k}^{2}$. The maximum eigenvalue $\hat{\lambda}_{\zeta}$ of $\Delta_{1} \oplus \Delta$ is the maximum eigenvalue of $\Delta_{1}:=0$ plus the maximum eigenvalue of $\Delta:=\xi_{1}$; that is, $-\xi_{2}:=-4 \sin ^{2} \frac{s \pi}{2 n}=-\xi_{1}$. Let $\lambda_{M}$ and $\lambda_{m}$ be the maximal and the minimal Lyapunov exponents over $M_{k}^{2} \subset M_{1}^{2}$. Then from the definition of Lyapunov type numbers in $\S 2$, we see that

$$
\alpha\left(z_{0}\right)=-k \xi_{1}+\lambda_{M} \text { and } \beta\left(z_{0}\right)=\frac{\lambda_{M}-k \xi_{1}}{\lambda_{m}} .
$$

Thus by Lemma 2, the synchronization manifold $M_{k}^{2}$ is robust if $k$ satisfies the inequality

$$
k>\max \left\{\frac{1}{\xi_{1}}\left(\lambda_{M}, \lambda_{M}-\lambda_{m}\right)\right\} .
$$

Taking $k_{0}=\max \left\{\frac{1}{\xi_{1}}\left(\lambda_{M}, \lambda_{M}-\lambda_{m}\right)\right\}$ completes the proof.

\section{Conclusion}

We have observed that persistence of the synchronization manifold crucially depends on the relationship between the dissipativeness of the individual oscillators and the coupling strength. There is a critical value of the coupling strength, $k_{0}$, above which the robustness of the synchronization manifold is guaranteed. The synchronization manifold needs to be normally hyperbolic and invariant in order for it to be robust. The proof 
for the condition of robustness depends on the coupling configuration and persistence of normal hyperbolicity. This work can easily be extended to three dimensional cases.

\section{References}

AFRAIMOVICH, V.S., BYKOV, V. and SHILNKOV, L. 1983. On Structurally Unstable Attracting Limit sets of Lorenz attractor Type, \{lit Tran. of Moscow Math. Soc. 44:153-216.

CAHN, J.W. 1960. Theory of growth and interface motion in crystalline material, Acta Metallurgica 8: 554-562.

CHOW, S.N., LIU, W. 1997. Synchronization, stability and normal hyperbolicity. Resenhas IME-USP. 3: 139158.

CUOMO, K.M. and OPPENHEIM, A.V. 1992. Synchronized Chaotic Circuits and systems for communications, MIT Research Laboratory of electronics technical report $\mathbf{5 7 5}$ (unpublished).

CUOMO, K.M., OPPENHEIM, A.V., 1993. Circuit implementation of synchronized chaos with applications to communications, it Phys. Rev. Lett. 71(1): 65-68.

CUOMO, K.M., OPPENHEIM, A.V. 1993. Chaotic signals and systems for communication, ICASSP 3: 137140.

CHUA, L.O. and ROSKA, T. 1993. The CNN Paradigm. IEEE Trans. Circuits Syst., 40: 147-156.

FENICHEL, N. 1971. Persistence and smoothness of invariant manifolds for flows. Ind., Univ. Math. J., Vol. 21 3: $193-225$.

FUJISAKA, H., YAMADA, T. 1983. Stability theory of synchronized motion in coupled-oscillators Systems. Prog. Theor. Phys. 69: 32-47.

HALE, J. 1997. Diffusive coupling, dissipation and synchronization J. Dyn. Differ. Equ. 9: 1-52.

HIRSCH, M.W., PUGH, C.C., SHUB, M. 1977. Invariant manifolds, Lecture Notes in Mathematics Vol 583. Springer-Verlag: Berlin, Heidelberg, New York.

JOSIĆA, K. 2000. Synchronization of chaotic systems and invariant manifolds, Nonlinearity 13:1321-1336.

LANCASTER, P. 1985. Tismenetsky, M.; The theory of Matrices, Academic press.

MANÉ, R. 1977. Persistent manifolds are normally hyperbolic, Trans. Amer. Math. Soc. 246: 261-283.

WASIKE, A.A.M. 2003. Synchronization and oscillator death in diffusively coupled lattice oscillators International Journal of Mathematical science, 2(1): 67-82.

WIGGINS, S. 1994. Normally Hyperbolic Invariant Manifolds in Dynamical Systems; Springer-Verlag: Berlin, Heidelberg, New York.

Received 27 February 2007

Accepted 11 October 2007 